\title{
A influência da diversidade de gênero e da sociabilidade no desempenho acadêmico de equipes de estudantes de graduação em Computação - uma análise preliminar
}

\author{
Danilo Gazzoli Resende, Luiz Celso Gomes Jr , Rita Berardi \\ ${ }^{1}$ Departamento Acadêmico de Informática \\ Universidade Tecnológica Federal do Paraná (UTFPR) \\ Av. 7 de setembro 3165 - CEP 80230-901 - Curitiba - PR - Brazil \\ daniloresende@alunos.utfpr.edu.br, \{gomesjr,rita\}@dainf.ct.utfpr.edu.br
}

\begin{abstract}
For groups of students to function effectively, it is important to foster diversity, according to the Team-based Learning methodology (TBL). With this prerogative, this study aims to understand the relationship between academic performance and the context of gender diversity and sociability. The study was performed based on student teams of a Databases class in undergraduate courses in Computing. Data on social connections, gender, team formation and performance were aggregated for the analysis. Preliminary results suggest that more diverse teams tend to perform better. In terms of sociability, we identified that groups with more friends among themselves and those formed by more sociable individuals also tend to perform better.
\end{abstract}

Resumo. Para que equipes de alunos funcionem da maneira mais eficaz é importante que elas sejam as mais diversas possíveis de acordo com a metodologia Aprendizagem baseada em Times (TBL). Com esta prerrogativa, este estudo visa o entendimento da relação entre o desempenho acadêmico e o contexto da diversidade de gênero e sociabilidade. O estudo foi realizado em equipes de alunos de uma disciplina de Banco de Dados em cursos de graduação em Computação. Dados de conexões sociais, gênero, formação de equipes e desempenho foram agregados para a realização das análises. Os resultados preliminares sugerem que equipes mais diversas tendem a ter desempenho melhor. Em termos de sociabilidade, identificamos que as equipes com mais amigos entre si e os formados por indivíduos mais sociáveis tendem a ter melhor desempenho.

\section{Introdução}

De acordo com a teoria de aprendizagem social de Vygotsky [Vygotsky 1980], o sujeito desenvolve importante potencial de aprendizado ao interagir com o próximo. A formação de equipes em sala de aula oportuniza o aprendizado em diversas dimensões, tanto de aspectos técnicos quanto sociais. No entanto, para que a proposta produza os efeitos esperados, não basta colocar os estudantes agrupados em conjunto, é necessário observar vários fatores na efetividade da construção desta equipe. Um dos fatores essenciais para o sucesso da efetividade do trabalho em equipe é a diversidade dessa equipe, em termos de gênero, etnia, sociabilidade entre outros, conforme afirma a metodologia de Aprendizagem Baseada em Equipes (TBL) [Michaelsen and Sweet 2008]. 
Em cursos ligados às áreas STEM (Science, Technology, Engineering e Mathematics), a composição de equipes ganha uma característica delicada pela desequilibrada proporção de gênero. Nestes cursos a quantidade de meninas em sala de aula costuma ser bastante inferior à quantidade de meninos e em um primeiro momento, uma prática docente comum é buscar promover a diversidade nas equipes: de conhecimento, sociabilidade e gênero. No entanto, pelo número extremamente baixo de meninas, geralmente as equipes mais diversas são compostas em sua maioria por meninos e com 1 ou 2 meninas. Apesar de comum, estudos têm se concentrado em observar se esta dinâmica de composição de equipes influencia o desempenho acadêmico[Vieira et al. 2017, Hirsch et al. 2017, Hart 2016].

Outro fator estudado neste contexto está relacionado às questões sociais estabelecidas entre os membros da equipe. Muitas vezes ao serem solicitados que formem equipes, os estudantes tendem a buscar seus amigos nesta composição. Contudo, não é óbvia a relação de sucesso entre amizade e aprendizagem, neste contexto. A tendência é buscar mais uma vez, a diversidade no grau de sociabilidade entre os membros para evitar coalizões dentro da equipe que possuem potencial para prejudicar seu desempenho acadêmico, um dos princípios do TBL (Team Based Learning) [Michaelsen and Sweet 2008].

Na literatura internacional, o estudo sobre a diversidade de gênero na formação de equipes já produziu resultados muito relevantes no que diz respeito à temática na infância e adolescência, principalmente no que tange às mudanças sofridas pelas meninas nessas fases. Um exemplo clássico desta transformação é a diminuição da autoestima e satisfação com suas próprias habilidades a partir dos 9 anos em média [of University Women and the Analysis Group 1994]. Essa mudança na percepção da sua autoconfiança pode sinalizar muitos aspectos da vida acadêmica de meninas que podem inclusive impactar na sua escolha e permanência em cursos majoritariamente frequentado por meninos. Sendo assim, faz-se necessário que se busquem cenários para avaliar a influência de gênero e sociabilidade na composição de equipes para o desempenho acadêmico. Apesar desta demanda, avaliar a trajetória de meninos e meninas da educação brasileira é um desafio principalmente devido à ainda restrita produção acadêmica sobre as desigualdades educacionais sob a ótica de gênero [Sousa 2017]. Somado a isto, faz-se necessário desenvolver estudos não somente no contexto acadêmico infantil, mas também no ensino superior, principalmente quando este estiver na área STEM na qual o desafio de evitar evasão é mais prevalente.

Este estudo apresenta resultados preliminares de uma análise sobre a influência da diversidade de gênero e do grau de sociabilidade em equipes de uma disciplina de Banco de Dados ofertada em dois cursos de graduação (Sistemas de Informação e Engenharia de Computação). Os dados foram coletados durante a atividade prática proposta para avaliação dos alunos (Seção 3). As análises sugerem que o desempenho acadêmico das equipes tende a melhorar quando a diversidade aumenta (Seção 4.1). Em termos do grau de sociabilidade, identificamos que meninas tendem a reportar mais conexões de amizade. Sobre a influência dos laços sociais no desempenho das equipes, as análises sugerem que aquelas formadas por pessoas que se conheciam anteriormente tendem a ter melhores resultados, assim como aquelas formadas por indivíduos mais sociáveis (Seção 4.2). 
IX Congresso Brasileiro de Informática na Educação (CBIE 2020)

Anais do XXXI Simpósio Brasileiro de Informática na Educação (SBIE 2020)

\section{Trabalhos relacionados}

A formação de equipes de estudo e o desempenho dos seus membros é fonte de discussões em trabalhos acadêmicos. Para Tardeli et al. [Tardelli et al. 2019], equipes organizadas tanto tecnicamente quanto emocionalmente podem resultar em soluções mais próximas do desejável, através de uma análise de seus componentes em um viés psicológico a fim de formar equipes que possuam maior compatibilidade com as necessidades da tarefa a ser cumprida. Entre os fatores relevantes na análise da qualidade da formação de grupos, destacamos aqui a diversidade de gênero e de sociabilidade dos alunos membros das equipes.

Um dos fatores importantes no desempenho de alunos é o ciclo social. Gomes [Gomes Jr 2019] avalia os dados de notas e círculos de amizades em turmas e seus resultados apresentam uma associação relevante entre fatores sociais e desempenho acadêmico. Além disso, a análise indica que alunos com maior capital social tendem a obter melhores rendimentos bem como aqueles que possuem amigos com boas notas tendem a se recuperar mais facilmente de notas baixas. Igualmente, Gašević et al. [Gašević et al. 2013] discutem a relação entre os vínculos sociais e o desempenho acadêmico ao formular a hipótese de que esta determinada performance é positivamente associada ao capital social acumulado durante o ciclo acadêmico.

Castilho et al. [Castilho et al. 2014] verificam a relação entre formação de equipes e a interação de seus membros em uma rede social e conclui que as equipes se constituem baseando-se em proxies derivados do Facebook. Neste sentido, esta rede social intermedeia conexões, algo que justifica a força de vínculos, popularidade e homofilia em vez de levar em consideração o histórico de notas [Castilho et al. 2014].

Questões relacionadas com o gênero também estão sendo muito analisadas em ambientes acadêmicos, sobretudo em áreas historicamente majoritariamente masculinas. Em [Vieira et al. 2017] é apresentada uma comunidade criada e frequentada exclusivamente por mulheres na área da tecnologia motivada principalmente pela sensação de ter a capacidade questionada em equipes formadas por homens e mulheres. $\mathrm{O}$ trabalho discute sobre o conforto percebido pelas mulheres em comunidades com público restritamente feminino. Por meio de um questionário respondido por 82 mulheres com idade entre 18 e 34 anos com ensino superior ou em andamento, foi possível notar o maior conforto em equipes restritas a mulheres.

Hirsh et al. [Hirsch et al. 2017] compararam o desempenho de várias meninas do Middle School (equivalente ao Ensino Fundamental II no Brasil) após a participação em cursos de verão sobre engenharias. A comparação aconteceu entre meninas que participaram de equipes exclusivas para meninas com meninas que participaram de equipes mistas. Os resultados indicam que meninas em equipes exclusivas de meninas, ao final do curso demonstraram melhor compreensão sobre a profissão na área de STEM, quais as responsabilidades e como é a rotina desses profissionais. Também foi possível notar uma visão mais inclusiva dessas meninas com relação à possibilidade de uma mulher também ser uma profissional dessas áreas.

Por outro lado, Friend [Friend 2006] também executou um experimento comparando o desempenho de estudantes do Middle School em aulas de ciências, agrupados por gênero. Neste estudo, os resultados indicam que não houve diferenças significativas no 
IX Congresso Brasileiro de Informática na Educação (CBIE 2020)

Anais do XXXI Simpósio Brasileiro de Informática na Educação (SBIE 2020)

desempenho acadêmico dos estudantes do mesmo gênero.

A diversidade de gênero é um fator importante nos mais diversos contextos da sociedade. Botella et al. [Botella et al. 2019] discutem a importância e os benefícios da participação feminina no setor de tecnologia. As autoras descrevem um programa de estímulo à participação feminina em diversos cursos de STEM. Um dos pontos centrais levantados é o incentivo a atividades que integrem as meninas no ambiente acadêmico.

Os benefícios da participação de meninas em atividades de equipe estão bem estabelecidos. Curseu et al. [Curşeu et al. 2018] acompanharam o desempenho de 118 equipes de alunos em um curso de primeiro ano de graduação. Os autores demonstraram que uma parte significativa da contribuição de meninas no desempenho dos equipes é intermediada por uma maior qualidade nas discussões dos grupos.

A análise apresentada neste artigo considera todos estes aspectos discutidos acima. Nosso objetivo é estudar como estas questões se manifestam conjuntamente num contexto de cursos de graduação em computação.

\section{Coleta e processamento de dados}

Os dados foram coletados a partir de turmas de alunos graduandos dos cursos de Sistemas de Informação e Engenharia da Computação de uma universidade federal. As disciplinas foram oferecidas semestralmente entre 2017 e 2019.

O processo de coleta foi intermediado pela aplicação de um trabalho prático em sala de aula nas disciplinas de Bancos de Dados, no qual o professor solicitou que os alunos implementassem e analisassem uma rede social da turma. O trabalho foi proposto no início do semestre, quando os alunos registraram seus dados de conexões de amizades entre os participantes da disciplina em uma aplicação disponibilizada pelo professor, com a ciência dos alunos de que os dados seriam usados em futuras análises como a deste artigo. Uma verificação foi executada a fim de garantir que houvesse a reciprocidade entre as conexões de amizade reportadas e aquelas amizades não recíprocas foram transformadas em recíprocas.

Para a análise, os dados de conexões sociais foram então agregados aos dados de desempenho acadêmico. Os dados utilizados foram: equipes formadas para a implementação do trabalho prático, notas individuais de provas, notas do trabalho de implementação da rede social e notas finais. As equipes se formaram livremente, sem orientação do professor para a escolha de integrantes. O tamanho esperado das equipes é de 3 alunos, mas há uma pequena variação por conta de divisão com resto e desistências.

Os dados dos alunos desistentes não foram considerados na análise, resultando em total de 186 alunos participantes na análise, dos quais 164 são meninos e 22 meninas, distribuídos em 7 turmas: 3 em 2017, 2 em 2018 e as demais em 2019 (Figura 1).

Nos dados dos alunos não havia registro sobre gênero. Para possibilitar a análise, foi utilizada uma biblioteca disponibilizada em [Álvaro Justen 2010] que provê uma API (Application Programming Interface) criada com base no banco de dados do censo do IBGE (Instituto Brasileiro de Geografia e Estatística) de 2010. Para determinar o gênero, a API usa a frequência pré-existente do nome pesquisado para os gêneros masculino e feminino como critério de classificação. 


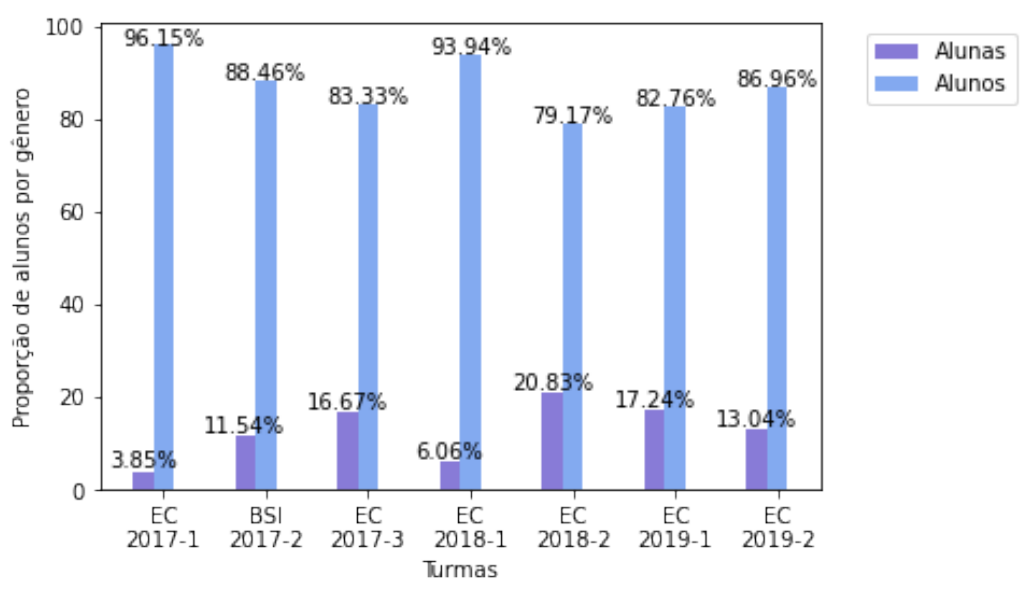

Figura 1. Distribuição de alunos e alunas por semestre.

\section{Análise de dados e discussões}

Com o objetivo de compreender as relações entre sociabilidade e diversidade de gênero com o desempenho acadêmico nos contextos de equipe, empregamos uma análise exploratória. Os gráficos produzidos pela análise são acompanhados de medições estatísticas para quantificar as associações identificadas.

A baixa participação de meninas nos cursos de computação nesta universidade (13\% dos alunos) dificulta a obtenção de resultados estatisticamente relevantes e por isso as tendências identificadas a seguir devem ser consideradas com cautela. O objetivo é iniciar uma discussão sobre as questões e estimular mais pesquisas sobre o assunto no contexto da computação brasileira.

\subsection{Estudo da diversidade e o desempenho acadêmico}

Como mencionado acima, os cursos de computação são predominantemente compostos por meninos. Neste sentido, esta primeira análise buscou entender a influência da diversidade de gênero no desempenho acadêmico, especialmente no contexto das dinâmicas das equipes.

\subsubsection{Diversidade e desempenho acadêmico}

Em um primeiro momento, esta análise foi conduzida a fim de verificar se a participação de meninas influencia no aumento das notas nas equipes. Para isto, comparamos as médias obtidas nos trabalhos em equipes com e sem meninas. Desta forma, as equipes que possuíam alguma participação feminina foram separadas daquelas contendo somente participação masculina.

Na Figura 2 são apresentados dois gráficos que relacionam as notas de trabalho a equipes com e sem meninas. Como exibido no gráfico de caixa (Figura 2 (a)), visualmente as equipes com participação feminina apresentam uma menor dispersão e uma mediana ligeiramente superior. No gráfico da Figura 2(b), os mesmos dados são demonstrados em uma outra visão, na qual ficam evidentes as concentrações dos valores das notas, de maneira não linear. 
A média para a nota do trabalho para as equipes com alguma participação feminina é 8.584, ao passo que equipes sem meninas obtiveram a média de 8.268. A diferença das médias não alcança relevância estatística (valor-p $=0.405)$ e o intervalo da diferença das médias entre - 0.44 e 1.07 (95\% de confiança) indica que amostragens alternativas poderiam produzir resultados diferentes. Esta é, porém, uma indicação de que meninas podem contribuir positivamente no desempenho das equipes, que reforça descobertas anteriores nas pesquisas sobre metodologia TBL [Michaelsen and Sweet 2008].

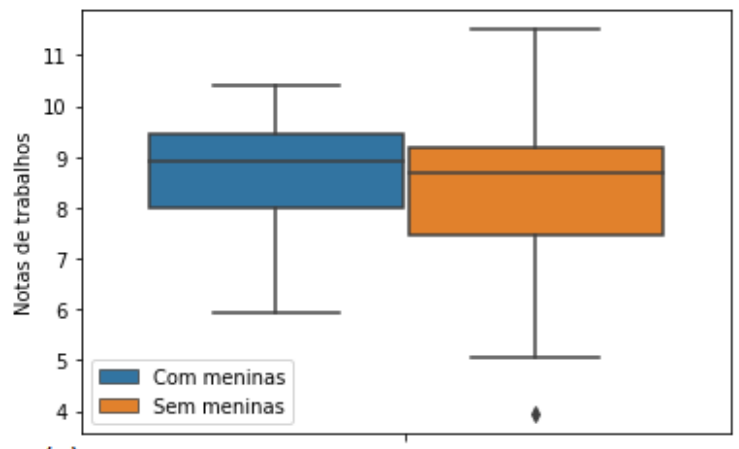

(a)

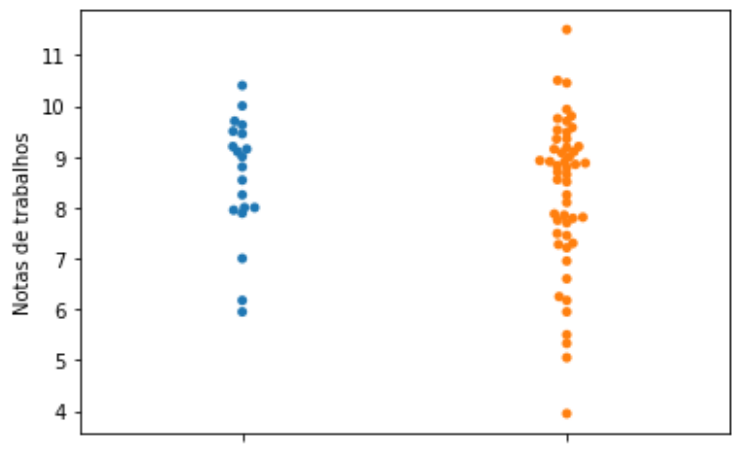

(b)

Grupos com meninas e sem meninas

Figura 2. "Nota de trabalho" relacionada a equipes com meninas e sem meninas em duas visões: (a) um gráfico de caixa e (b) um gráfico de enxame com a concentração dos dados de maneira não linear.

Para compreender melhor o relacionamento entre a presença de meninas e o desempenho das equipes, fizemos também uma análise de correlação entre a nota do trabalho e a proporção de meninas na equipe (Figura 3). A Figura 3 distribui as equipes de acordo com porcentagem de meninas e nota do trabalho. Nesta análise, o método de Pearson produziu uma correlação no valor de 0.076 , (valor-p $=0.527$ ), indicando uma pequena influência positiva, com baixa significância estatística. Assim como o resultado anterior, apesar da baixa significância estatística, as análises sugerem que equipes com meninas tendem a ter um desempenho melhor.

Para entender se a influência positiva da presença de meninas poderia ser explicada pelo nível de desempenho individual delas, analisamos também as notas de provas de meninos e meninas. A Figura 4 ilustra a concentração de notas nos quartis e as suas medianas. Um fato interessante demonstra que a mediana das alunas é superior à dos alunos porém as suas médias descrevem o oposto, sendo 6.29, com intervalo de (5.61, $6,97)$ e nível de confiança de $95 \%$ para elas e 6.69 , com intervalo de $(6.44,6.94)$ para eles (95\% de confiança). Apesar da baixa relevância estatística entre as notas de provas e gêneros (valor-p $=0.27$ ), a análise sugere que a influência positiva da presença de meninas nas equipes não pode ser explicada simplesmente por melhores desempenhos individuais. Este aspecto está de acordo com os resultados de Curseu et al. [Curşeu et al. 2018], que enfatizam a importância de meninas na intermediação das interações na equipe.

Uma última análise foi conduzida com o intuito de entender se a diversidade pode ser benéfica no contexto geral das turmas, i.e. se há relação entre desempenho acadêmico médio e a quantidade de meninas nas turmas. Para tanto, a proporção de gênero em cada turma foi relacionada com as variáveis "média das provas" e "média dos trabalhos" respectivamente, produzindo os gráficos ilustrados na Figura 5. 


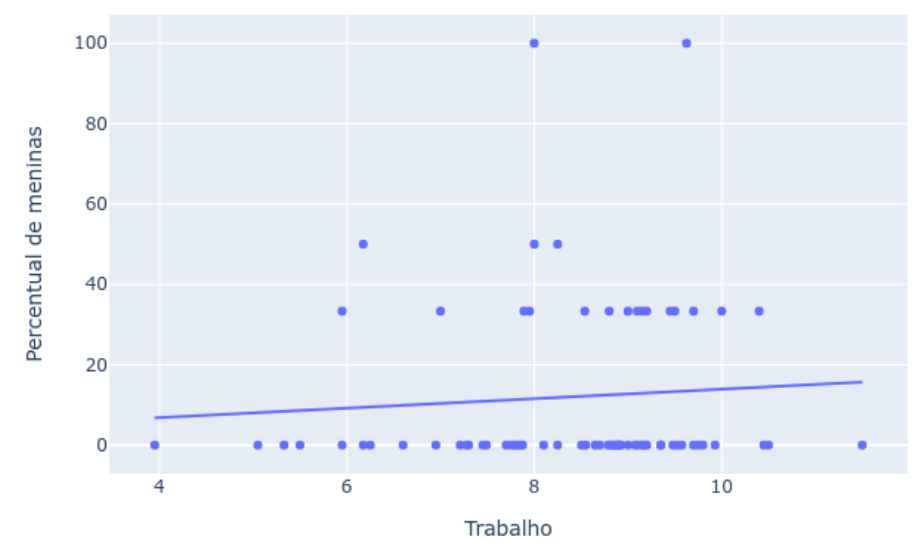

Figura 3. "Nota de trabalho" relacionada com a proporção de meninas nas equipes.

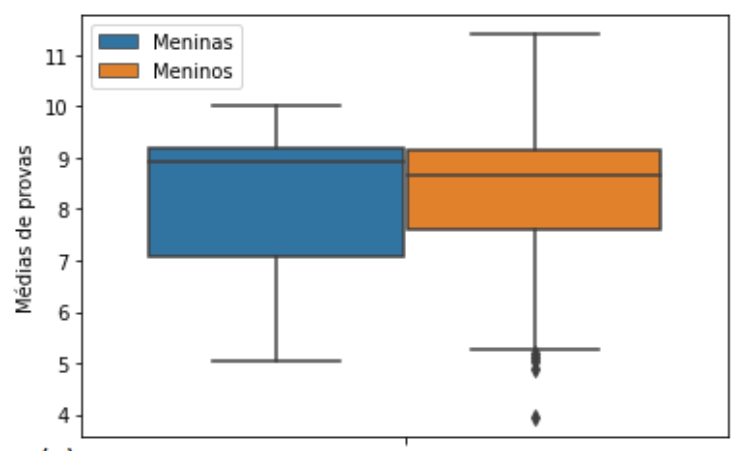

(a)

Meninas e Meninos

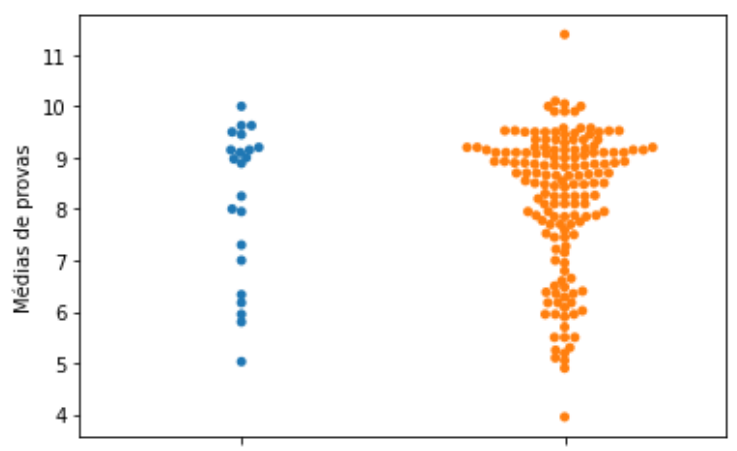

(b)

Meninas Meninos

Figura 4. "Notas de provas" relacionadas a meninas e meninos no contexto geral em duas visões: (a) um gráfico de caixa e (b) um gráfico de enxame com a concentração dos dados de maneira não linear.

Os valores de correlação de Pearson também foram computados relacionando as variáveis com a proporção de meninas. Para a variável "média dos trabalhos", a correlação obteve 0.0826 , valor-p igual a 0.86 (Figura 5 (b). A variável "média das provas" obteve a correlação de -0.697 , com valor-p igual a 0.082 (Figura 5 (a).

Embora a presença de meninas beneficie as notas das equipes, esta análise demonstrou uma correlação alta e negativa no contexto da turma e as notas de prova, sugerindo que neste contexto turmas mais diversas tendem a ter notas mais baixas. $\mathrm{O}$ baixo número de turmas $(\mathrm{n}=7)$ dificulta a obtenção de resultados estatisticamente significativos, mas a análise dos gráficos (Figura 5) evidencia esta tendência. É importante realizar mais análises para identificar potenciais variáveis de confusão que poderiam ajudar a evidenciar as associações pertinentes.

\subsection{Estudo da sociabilidade e o desempenho acadêmico}

Como relatado nas seções anteriores, a interação social é um fator preponderante na execução dos trabalhos, visando o esclarecimento de ideias e o aporte de conhecimento. 

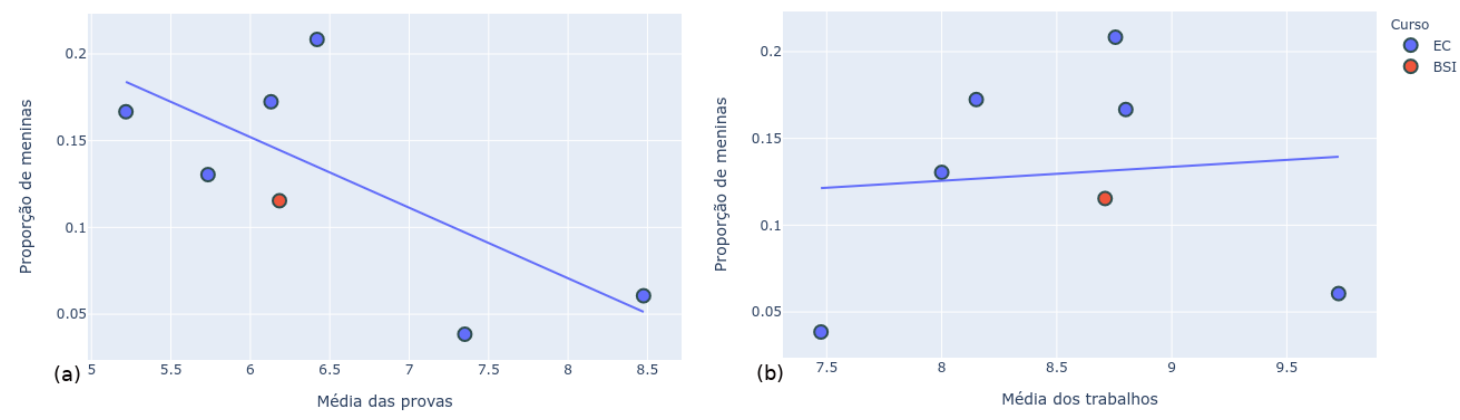

Figura 5. Média das provas(a) e média de trabalhos (b) associados à proporção de alunas.

Levando isto em consideração, esta análise buscou entender a influência da diversidade de sociabilidade e a sua contribuição no desempenho acadêmico das equipes.

A partir dos dados respondidos na rede social do trabalho proposto para os alunos (na qual cada estudante indicou suas conexões de amizade), buscou-se entender se há alguma diferença de sociabilidade, em termos de capital social, entre meninos e meninas. Para esta pesquisa, entende-se como capital social o número total de ligações de amizade de um aluno.

No quesito sociabilidade, os alunos apresentaram um capital social médio de 6.87 (intervalo $=(6.14,7.61)$, confiança $=95 \%)$, ao passo que as alunas obtiveram 8.09 (intervalo $=(6.28,9.89)$, confiança $=95 \%)$. Apesar da baixa significância estatística (valor-p = $0.24)$, os resultados sugerem que meninas são mais sociáveis do que meninos.

A rede social também foi explorada dentro do contexto das equipes a fim de verificar se equipes formadas por membros amigos entre si tendem a obter uma nota maior. Ao analisar a correlação entre a proporção de amigos e a nota de trabalho da equipe, obtivemos 0.25 (valor-p = 0.033), o que demonstrou uma correlação positiva e significativa entre o rendimento acadêmico e o capital social, indicando que equipes formadas por membros que são amigos entre si (e não somente colegas nesta turma) tendem a ter um desempenho acadêmico melhor.

De maneira análoga, o capital social foi analisado visando entender se equipes de alunos mais sociáveis têm melhor desempenho. Ao verificar a correlação entre sociabilidade média (i.e. média dos contatos dos participantes da equipe) com a nota de trabalho da equipe, esta correlação resultou em 0.39 , resultado estatisticamente significativo (valor-p $=0.0006)$.

Para as duas últimas análises, foi evidenciada uma correlação positiva entre o capital social e a nota de trabalho da equipe (Figura 6). Estes resultados indicam que equipes mas entrosadas e mais sociais tendem a ter melhores resultados.

\section{Discussões e conclusão}

A metodologia de Aprendizagem baseada em Times (TBL) incentiva os princípios da educação baseada em equipes e de maneira diversa. Com esta premissa, este trabalho buscou entender a influência da diversidade de gênero e da sociabilidade no desempe- 

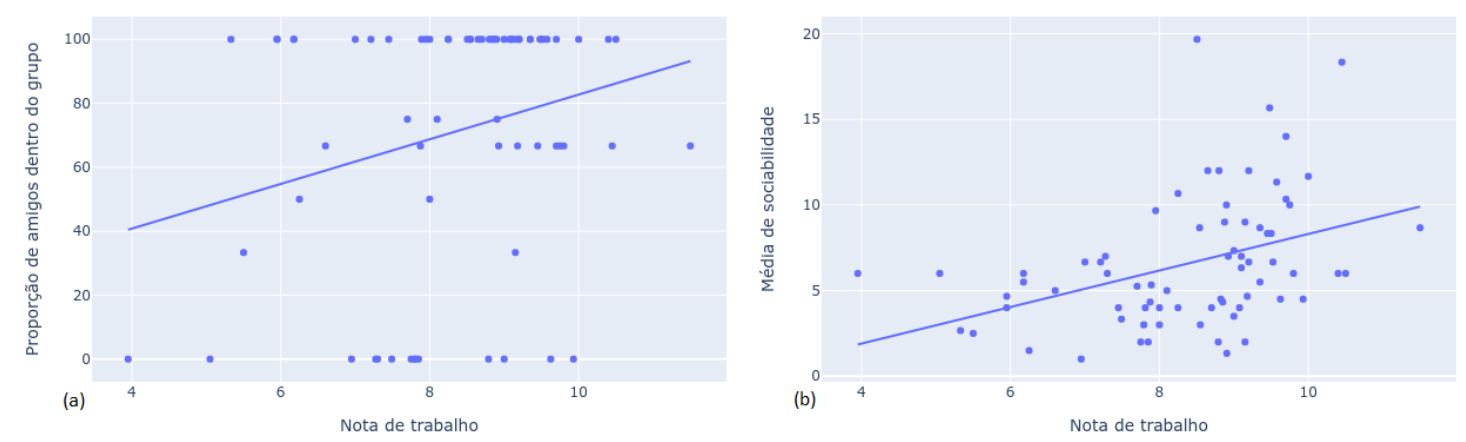

Figura 6. Nota de trabalho e sociabilidade "intra-equipe" (a) e "intra-turma" (b)

nho acadêmico da disciplina Banco de dados em cursos de Computação (Engenharia de Computação e Sistemas de Informação) de uma universidade pública brasileira.

Após a avaliação das características em 7 diferentes turmas, pôde-se perceber que os resultados indicaram que, apesar da baixa significância estatística, a participação de meninas nas equipes eleva o desempenho acadêmico. Em contraposição, os resultados indicam que meninos obtêm notas melhores em provas do que meninas, o que nos direciona à necessidade de entender a diferença de desempenho entre prova e trabalho de acordo com o gênero a fim de averiguar se meninas tendem a se adaptar melhor a trabalhos práticos e com isso, reavaliarmos o peso que é dado às provas na maioria das disciplinas para o cálculo da nota final de cada discente.

Os resultados demonstraram que há uma forte indicação de que conhecer pessoas na mesma equipe e ser sociável com os demais colegas da turma conduzem a um bom desempenho acadêmico, uma vez que esta determinada prática incentiva a troca de ideias e contribuições nos trabalhos. Desta forma, a sociabilidade corrobora de forma análoga com as notas, sendo tal característica também percebida em outros estudos.

Com relação à sociabilidade, percebe-se que esta característica é mais latente em meninas do que meninos. Este quesito pode explicar o benefício identificado na presença de meninas noas equipes. O modelo precisa ser aperfeiçoado para uma análise mais conclusiva.

Estes achados são importantes para contribuir com o debate e chamar a atenção de gestores, coordenadores e docentes sobre a relevância da diversidade e a sociabilidade no ambiente educacional. No tocante aos discentes, esta pesquisa também traz importantes contribuições pois, tendo em mente o cenário que propicia as discussões e os possíveis impactos em sua performance acadêmica, é possível que eles passem a buscar uma melhor interação para obter um ambiente de aprendizagem mais inclusivo e produtivo.

As associações negativas entre participação de meninas e notas médias das turmas precisa ser investigada em detalhes. Para essas análises futuras planejamos incluir mais variáveis para análise e incluir dados de mais disciplinas e diferentes professores. 
IX Congresso Brasileiro de Informática na Educação (CBIE 2020)

Anais do XXXI Simpósio Brasileiro de Informática na Educação (SBIE 2020)

\section{Referências}

Botella, C., Rueda, S., López-Iñesta, E., and Marzal, P. (2019). Gender diversity in stem disciplines: A multiple factor problem. Entropy, 21(1):30.

Castilho, D., de Melo, P. V., Quercia, D., and Benevenuto, F. (2014). Working with friends: Unveiling working affinity features from facebook data. In ICWSM 2014.

Curşeu, P. L., Chappin, M. M. H., and Jansen, R. J. G. (2018). Gender diversity and motivation in collaborative learning groups: the mediating role of group discussion quality. Social Psychology of Education, 21(2):289-302.

Friend, J. (2006). Research on same-gender grouping in eighth grade science classrooms. RMLE Online, 30(4):1-15.

Gašević, D., Zouaq, A., and Janzen, R. (2013). “choose your classmates, your gpa is at stake!": The association of cross-class social ties and academic performance. American Behavioral Scientist, 57(10):1460-1479.

Gomes Jr, L. (2019). In-class social networks and academic performance: how good connections can improve grades. In Anais Principais do XXXIV Simpósio Brasileiro de Banco de Dados, pages 25-36. SBC.

Hart, L. C. (2016). When "separate" may be better: Exploring single-sex learning as a remedy for social anxieties in female middle school students. Middle School Journal, 47(2):32-40.

Hirsch, L. S., Berliner-Heyman, S., Cano, R., and Cusack, J. L. (2017). The effectiveness of single-gender engineering enrichment programs: A follow-up study. In American Society for Engineering Education Zone II Conference, San Juan, PR.

Michaelsen, L. K. and Sweet, M. (2008). The essential elements of team-based learning. New directions for teaching and learning, 2008(116):7-27.

of University Women, A. A. and the Analysis Group, G.-L. (1994). Shortchanging Girls, Shortchanging America: Executive Summary: a Nationwide Poll that Assesses Selfesteem, Educational Experiences, Interest in Math and Science, and Career Aspirations of Girls and Boys Ages 9-15. Amer Assn of Univ Women.

Sousa, A. P. d. (2017). Desigualdades nas trajetórias entre meninos e meninas nos anos finais do ensino fundamental da ride-df: uma análise dos dados do censo escolar 20122016.

Tardelli, A., França, J., Dias, A., Vivacqua, A., and Borges, M. (2019). A influência da personalidade do aluno na construção de grupos de trabalho em sala de aula. In Brazilian Symposium on Computers in Education), volume 30, page 1916.

Vieira, C. C., Soares, A. C. R., and Ribeiro, S. F. (2017). Incentivos à igualdade de gênero: estudo de caso de uma comunidade formada somente por mulheres da área de tecnologia. In Anais do XI Women in Information Technology. SBC.

Vygotsky, L. S. (1980). Mind in society: The development of higher psychological processes. Harvard university press.

Álvaro Justen (2010). Classificação de gênero em nomes brasileiros, com base em dados do censo de 2010. https : / / www. brasil. io. Accessed: 2020-06-30. 